\title{
Modeling the Kinematics and Dynamics of Compliant Contact
}

\author{
Vincent Duindam Stefano Stramigioli \\ (v.duindam@ieee.org) (s.stramigioli@ieee.org) \\ Drebbel Institute for Mechatronics \\ University of Twente, The Netherlands
}

\begin{abstract}
In this paper, we discuss the modeling of the kinematics and dynamics of compliant contact between bodies moving in Euclidean space. First, we derive the kinematic equations describing the motion of the contact point when two rigid bodies are rolling on each other. Secondly, we extend these results to describe the motion of the closest points between two rigid bodies moving freely in space. Then, we use these results to model compliant contact between bodies, using a spatial spring and a damper to model energy stored and dissipated during contact.
\end{abstract}

\section{Introduction}

In many models of walking machines (e.g. $[1,2,3]$ ), the contact between a foot and the ground is modeled as a discontinuous change of velocity of the foot, and instant dissipation of energy. This type of contact model can be used to accurately describe contact with a flat, hard surface. However, when the soil is something softer or more curved (such as sand, mud and rocks, typically encountered in outdoor and space applications), a more detailed contact model is needed to capture the compliance and the shape of the contact in order to be able to analyze the stability and robustness of the walking robot.

In this paper, we look at the modeling of this type of contact. More specifically, we look at the kinematic aspects of compliant contact. The dynamic aspects are also briefly discussed, but we refer to [4] for the details.

The kinematic analysis consists of two parts: First, regular contact kinematics describes the movement of the contact point between two rigid bodies as the bodies roll and slide over each other. In other words, it describes the velocity of two points (one on each body) that have zero distance. Second, generalized contact kinematics describes the movement of two points (one on each body) that have the smallest distance between them. Both regular and generalized contact kinematics are important in the modeling of compliant contact, as contact can be lost when two objects bounce off each other.

The kinematics of regular contact have been modeled before $[5,6]$, and recently these results have been extended to the generalized kinematics case [7]. Both approaches however rely on an orthogonal parameterization of the surfaces, and they need the definition of certain matrices and extra contact coordinates, which mystify the resulting equations and make it much harder to get a physical interpretation.

Contrary to the results mentioned before, we do not use a coordinate-based approach in this paper, so the results hold for any parameterization of the surfaces (not only orthogonal parameterizations), and we do not require the introduction of special extra coordinates and matrices. It thus gives a simpler, intuitive idea of the contact kinematics, and the equations are still physically interpretable and not just mathematical results. We used the results of this paper in a contact model based on the Port-Controlled Hamiltonian approach [8].

This paper is organized as follows: first, Section 2 provides the necessary mathematical preliminaries and notation about the dynamics of rigid bodies and about their surfaces. Then, Section 3 discusses the kinematics of rigid bodies in point contact, and Section 4 extends the results to the kinematics of rigid bodies moving freely in space. Section 5 briefly discusses the use of the kinematic equations in a lumped model of compliant contact between two bodies, and Section 6 shows simulations of this contact model. Finally, Section 7 presents the conclusions and an outlook on future research.

\section{Preliminaries and Notation}

\subsection{Rigid Body Dynamics}

In this paper, we deal with rigid bodies moving in the Euclidean space $\mathcal{E}$, which means we can describe the position and orientation of every body by an element of the special Euclidean group $S E(3)$, once a reference frame has been chosen. As shown for example in $[9,10]$, elements of this group can be represented by a homogeneous matrix of the form

$$
H_{j}^{i}=\left[\begin{array}{cc}
R_{j}^{i} & p_{j}^{i} \\
0 & 1
\end{array}\right]
$$

where $R_{j}^{i}$ is a rotation matrix (element of the special orthonormal group $S O(3)$ ) and $p_{j}^{i}$ is a vector in $\mathbb{R}^{3} . H_{j}^{i} \mathrm{de}$ notes the change of coordinates from a right-handed coordinate frame $\Psi_{j}$ to another right-handed coordinate frame $\Psi_{i}$ and can thus be used for example to describe the position and orientation of a body (with attached coordinate frame $\Psi_{j}$ ) relative to a reference (inertial) coordinate frame $\left(\Psi_{i}\right)$.

The instantaneous velocity of a body $i$ with frame $\Psi_{i}$ relative to a body $j$ with frame $\Psi_{j}$ can be represented by a twist $T_{i}^{k, j}$, with

$$
T_{i}^{k, j}=\left[\begin{array}{c}
\omega_{i}^{k, j} \\
v_{i}^{k, j}
\end{array}\right]
$$


where $\omega_{i}^{k, j}$ denotes the angular velocity of body $i$ relative to body $j$ expressed in coordinate frame $\Psi_{k}$, and $v_{i}^{k, j} \mathrm{de}$ notes the instantaneous velocity (relative to frame $\Psi_{j}$ ) of the point fixed in frame $\Psi_{i}$ that passes through the origin of frame $\Psi_{k}$. A twist can be regarded as the derivative of a homogeneous matrix in the following way, using what is called a right translation of a Lie group [11]:

$$
\tilde{T}_{i}^{j, j}:=\left[\begin{array}{cc}
\tilde{\omega}_{i}^{j, j} & v_{i}^{j, j} \\
0 & 0
\end{array}\right]=\dot{H}_{i}^{j} H_{j}^{i}
$$

where $\tilde{\omega}=-\tilde{\omega}^{T}$ is the matrix equivalent to $(\omega \times \cdot)$.

We can also define a wrench $W_{i}^{k}$ (the dual of a twist), which describes the generalized forces acting on body $i$ and expressed in frame $\Psi_{k}$, as

$$
W_{i}^{k}=\left[\begin{array}{c}
\tau_{i}^{k} \\
F_{i}^{k}
\end{array}\right]
$$

where $F_{i}^{k}$ denotes the linear force and $\tau_{i}^{k}$ the momentum, acting on the point in the origin of frame $\Psi_{k}$. The dual product of a twist and a wrench (when expressed in the same coordinate frame) is equal to a power flow. More information on twists and wrenches can be found in $[9,10]$.

\subsection{Surface Description}

Consider a rigid body with a smooth, oriented surface $\mathcal{S}$, embedded in the Euclidean space $\mathcal{E}$. To this body, we rigidly attach a coordinate frame $\Psi$. In the frame $\Psi$, we can describe the surface (locally) as a bijective mapping $f: \mathcal{D} \rightarrow \mathcal{S}$, which assigns to each set of local coordinates $u \in \mathcal{D} \subset \mathbb{R}^{2}$ a point of the surface. The mapping $f$ is a (local) parameterization of the surface, and we assume this parameterization to be well-defined, i.e. the derivative mapping $f_{*}=\frac{\partial f}{\partial u}$ is continuous and has kemel zero, i.e. the partial derivatives $\frac{\partial f}{\partial u}$ are independent at all points.

At each point of the surface, we can find the unit vector $n(p)$ normal to the surface (we can compute this for example by taking the cross product between the partial derivatives of $f$ ). We can identify these unit vectors with points on the unit sphere $\mathbb{S}^{2}$, if we think of the point on the sphere as the tip of the normal vector with its base point in the center of the sphere.

The Gauss mapping $g: \mathcal{S} \rightarrow \mathbb{S}^{2}$ is defined as the mapping which takes a point $p$ on the surface and returns a point $g(p)$ on the sphere, corresponding to the unit normal at $p$. The smoothness and orientability of the surface ensure that the normal vector varies smoothly over the surface, and hence the mapping $g$ is smooth. This means that we can also define the derivative mapping $g_{*}: T \mathcal{S} \rightarrow T \mathbb{S}^{2}$. This derivative can be interpreted as follows: if we move tangent to the surface at velocity $\zeta \in T \mathcal{S}$, then the normal vector changes with velocity $g_{*} \zeta \in T \mathbb{S}^{2}$. Since the vector $g(p)$ is perpendicular to the surface at $p$ as well as to the sphere at $g(p)$, we can directly regard an element $g_{*} \zeta \in T_{g(p)} \mathbb{S}^{2}$ as an element $P g_{*} \zeta \in T_{p} \mathcal{S}$, where $P$ denotes the mapping from $T_{g(p)} \mathbb{S}^{2}$ to $T_{p} \mathcal{S}$.

The intuitive meaning of the differential $g_{*}$ of the Gauss map is curvature: the vector $g_{*} \zeta$ for some $\zeta \in T S$ describes the curvature of the surface when moving at velocity $\zeta$. If $g_{*}(p) \zeta=0$ for all $\zeta \in T_{p} \mathcal{S}$, then the surface is locally flat at $p$. If $\left\langle\zeta, P g_{*}(p) \zeta\right\rangle>0$ for all $\zeta \in T_{p} \mathcal{S}$, then we say that the surface is locally absolutely convex ${ }^{1}$ at $p$.

Figure 1 shows the relations between the various mappings and spaces. It is important to note that $f$ is a bijective mapping, hence it uniquely identifies coordinate-pairs to points on the surface and its derivative mapping $f_{*}$ is invertible. This means that although the equations in the following sections do not contain local coordinates $u$ or the surface parameterization $f$, we can always find coordinate expressions for these equations using $f, f_{*}$ and their inverses.

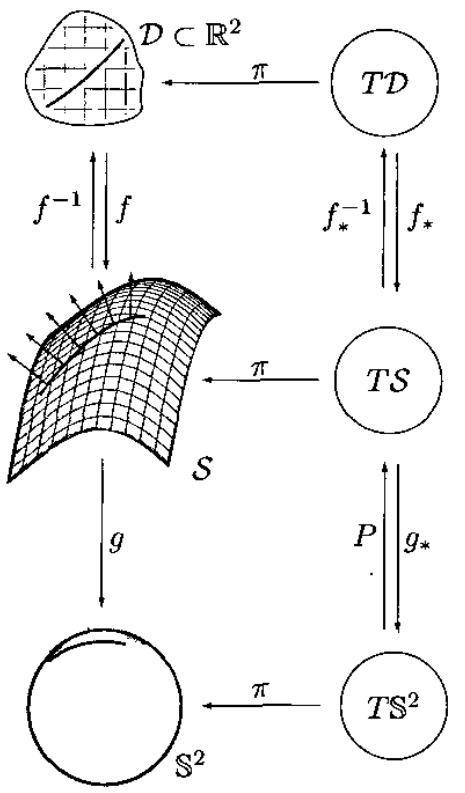

Figure 1: Relation diagram showing the mappings be tween the coordinate patch $\mathcal{D}$, the surface $\mathcal{S}$, the unit sphere $\mathbb{S}^{2}$ and their tangent spaces. The canonical projection $\pi$ is added for completeness; it takes an element $(p, \zeta)$ of the tangent bundle and returns its base point $p$.

\subsection{Numerical Notation}

Since we want to use the formal results in numerical simulation, we need ways to represent the geometrical ideas in vectors and matrices. If we use the formats denoted in Table 1, then the geometric equations in the following sections can be implemented directly into numerical equations.

For example, say that we want to compute the mapping $g_{*}$ and we only have a representation in local coordinates, i.e. a four-by-two matrix $\hat{g}_{*}: T \mathcal{D} \rightarrow T \mathbb{S}^{2}$ such that $\hat{g}_{*}=g_{*} f_{*}$. We can derive $g_{*}$ from this, but then we need $f_{*}^{-1}$. Now $f_{*}$ is represented by a four-by-two matrix (mapping the time derivative of local coordinates to the time derivative of a point in $\mathcal{E}$ ) and it cannot be inverted in the usual sense. However, if we take the definition given in

\footnotetext{
'We explicitly use the term 'absolutely convex' (which just means 'convex' in the usual sense) to distinguish it from the term 'relatively convex", which we defi ne in Section3
} 
the table (which is just the Moore-Penrose pseudo inverse), then the computation can be performed and $\hat{g}_{*} f_{*}^{-1}$ is a fourby-four matrix as required, mapping vectors $\dot{p} \in \mathbb{R}^{4}$ to vectors $g_{*} \dot{p} \in \mathbb{R}^{4}$ (of course, this mapping only has physical meaning for $\dot{p} \in T \mathcal{S}$ ).

Table 1: Numerical implementation of the geometrical equations. A diamond $(0)$ denotes an arbitrary number.

\begin{tabular}{|c|c|c|}
\hline type & example & numerical format \\
\hline local coordinates & 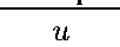 & {$\left[\begin{array}{ll}\diamond & \diamond\end{array}\right]^{T}$} \\
\hline point in $\mathcal{E}$ & $p$ & $\diamond 1]^{T}$ \\
\hline free vector in $\mathcal{E}$ & $\dot{p}$ & {$\left[\begin{array}{ll}0 & \diamond\end{array}\right.$} \\
\hline surface parameterization & $f(u)$ & {$\left[\begin{array}{ll}\diamond & \diamond\end{array}\right.$} \\
\hline Gauss map & $g(p)$ & $\diamond 0^{T}$ \\
\hline tangent mapping & $f_{*}$ & {$\left[\begin{array}{llll}\diamond & \diamond & \diamond & 0 \\
\diamond & \diamond & \diamond & 0\end{array}\right]^{T}$} \\
\hline inverse tangent mapping & $f_{*}^{-1}$ & $\left(f_{*}^{T} f_{*}\right)^{-1} f_{*}^{T}$ \\
\hline
\end{tabular}

\section{Regular Contact Kinematics}

We first consider the case of two rigid bodies in point contact, moving with a relative velocity represented by a twist $T_{1}^{2,2}=-T_{2}^{2,1}$ (this is exactly the case described by Montana [5]). We attach to each body $i$ a coordinate frame $\Psi_{i}$ and we assume to have a description $f_{i}$ of the surface of this body, expressed in frame $\Psi_{i}$.

If we then express the location of the point of contact as two points $p_{1}$ (expressed in frame $\Psi_{1}$ ) and $p_{2}$ (expressed in frame $\Psi_{2}$ ), then if the two bodies are in point contact, we have

$$
\left\{\begin{array}{l}
p_{1}=H_{2}^{1} p_{2} \\
g_{1}=-H_{2}^{1} g_{2}
\end{array}\right.
$$

where we abbreviated $g_{i}:=g_{i}\left(p_{i}\right)$. These equations just say that for point contact, the two contact points must be the same (when expressed in the same coordinate frame, in this case $\Psi_{1}$ ) and the normal vectors to the surfaces must be opposite. ${ }^{2}$

To obtain the kinematic equation relating the velocities of the contact points to the velocity of the bodies, we only need to take the time-derivative of (2) to obtain:

$$
\begin{aligned}
& \left\{\begin{array}{l}
\dot{p}_{1}=\dot{H}_{2}^{1} p_{2}+H_{2}^{1} \dot{p}_{2} \\
\dot{g}_{1}=-\dot{H}_{2}^{1} g_{2}-H_{2}^{1} \dot{g}_{2}
\end{array}\right. \\
& \left\{\begin{array}{c}
\dot{p}_{2}=H_{1}^{2} \dot{p}_{1}-H_{1}^{2} \dot{H}_{2}^{1} p_{2} \\
g_{1 *} \dot{p}_{1}=\tilde{T}_{2}^{1,1} g_{1}-H_{2}^{1} g_{2 *} \dot{p}_{2}
\end{array}\right.
\end{aligned}
$$

where we pre-multiplied (3) by $H_{1}^{2}$ to obtain an expression for $\dot{p}_{2}$. If we now substitute this expression into the second

\footnotetext{
${ }^{2}$ Note that we use a homogeneous matrix $H_{2}^{1}$ to change coordinates for points $\left(p_{2}\right)$ as well as for free vectors $\left(g_{2}\right)$. Normally, free vectors only need to be rotated (using the rotation part of the coordinate transformation), but since we express these vectors numerically as a four-by-one matrix with its last element zero, we can just as well use multiplication by the full homogeneous matrix.
}

line of (4) and repeat the whole derivation with objects 1 and 2 switched, we obtain the desired kinematic equations:

$$
\begin{aligned}
& \left(g_{1 *}+H_{2}^{1} g_{2 *} H_{1}^{2}\right) \dot{p}_{1}=\tilde{T}_{2}^{1,1} g_{1}-H_{2}^{1} g_{2 *} \tilde{T}_{1}^{2,2} p_{2} \\
& \left(g_{2 *}+H_{1}^{2} g_{1 *} H_{2}^{1}\right) \dot{p}_{2}=\tilde{T}_{1}^{2,2} g_{2}-H_{1}^{2} g_{1 *} \tilde{T}_{2}^{1,1} p_{1}
\end{aligned}
$$

Let us now briefly discuss the conditions under which these equations have unique solutions $\dot{p}_{1}, \dot{p}_{2}$. Because of the symmetry, we only consider the first equation, i.e. the equation for $\dot{p}_{1}$. Because the four-by-four matrix $\left(g_{1 *}+\right.$ $H_{2}^{1} g_{2 *} H_{1}^{2}$ ) has a non-zero kernel, we cannot simply invert this matrix and always get a unique result. Instead, we must look at the equation from a geometrical point of view.

First of all, since we look at the motion of the contact point over the surface, we must have $\dot{p}_{1} \in T_{p_{1}} \mathcal{S}_{1}$.

Secondly, since the domain of the mapping $g_{2 *}$ is $T_{p_{2}} \mathcal{S}_{2}$ and not all vectors in $\mathcal{E}$, we must have $\tilde{T}_{1}^{2,2} p_{2} \in T_{p_{2}} \mathcal{S}_{2}$. This constraint means that the velocity of the instantaneous contact point $\left(\tilde{T}_{1}^{2,2} p_{2}\right)$ can not have a component perpendicular to the surface, thus constraining the allowed relative motion to five degrees of freedom (which is clear from a physical point of view).

Finally, we need to ensure that a unique solution $\dot{p}_{1}$ exists for any twist satisfying the constraint above. Since both $\tilde{T}_{2}^{1,1} g_{1}$ and $H_{2}^{1} g_{2 *} \tilde{T}_{1}^{2,2} p_{2}$ are tangent to the surface, the codomain of the matrix $\left(g_{1 *}+H_{2}^{1} g_{2 *} H_{1}^{2}\right)$ must be the whole tangent plane to the surface, i.e. the matrix must have rank two. For physical reasons (no intersection of the surfaces) this means that the two surfaces must be relatively convex: the two non-zero eigenvalues of $\left(g_{1 *}+H_{2}^{1} g_{2 *} H_{1}^{2}\right)$ must be larger than zero. Physically, relative convexity means that if one surface is concave, then the other body must be extra convex. Absolute convexity (as defined in Section 2) can be considered as a special case: an absolutely convex surface is relatively convex to a plane.

\section{Generalized Contact Kinematics}

In this section, we extend the results of Section 3 to the more general case as depicted in Figure 2: we do not consider just the kinematics of the point of contact between the two bodies, but we look at the kinematics of the points on the surfaces which have the shortest (in the Euclidean sense) distance between them. We call this problem the generalized contact kinematics problem.

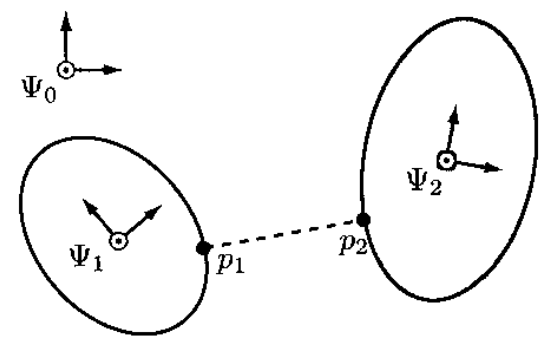

Figure 2: Two rigid bodies and their generalized contact points $p_{1}$ and $p_{2}$.

We use $p_{i}, i=1,2$ to denote the point on body $i$ expressed in frame $i$ such that the distance between $p_{1}$ and $p_{2}$ 
is the minimum distance between the bodies. This implies that the line connecting $p_{1}$ and $p_{2}$ must be perpendicular to both surfaces, which can be translated into the following equations:

$$
\left\{\begin{aligned}
p_{1}+\Delta g_{1} & =H_{2}^{1} p_{2} \\
g_{1} & =-H_{2}^{1} g_{2}
\end{aligned}\right.
$$

where $\Delta \in \mathbb{R}$ denotes the 'signed distance' between the generalized contact points:

$$
\Delta=\left\langle g_{1}, H_{2}^{1} p_{2}-p_{1}\right\rangle
$$

i.e. $\Delta>0$ means there is a distance $|\Delta|$ between the bodies, and $\Delta<0$ means the bodies have a maximum penetration distance of $|\Delta|$. The use of this definition for distance (instead of the usual $\left\|H_{2}^{1} p_{2}-p_{1}\right\|$ ) turns out to be very useful in the modeling of contact dynamics in Section 5.

Theorem 1 Given two rigid bodies and the generalized contact points as defined in (6). If the bodies are absolutely convex, then the velocity of the generalized contact points is uniquely determined by the following equations:

$$
\left\{\begin{array}{c}
\left(g_{1 *}+H_{2}^{1} g_{2 *} H_{1}^{2}\left(I+\Delta g_{1 *}\right)\right) \dot{p}_{2}= \\
\tilde{T}_{2}^{1,1} g_{1}+H_{2}^{1} g_{2 *}\left(\dot{\Delta} g_{2}-\tilde{T}_{1}^{2,2} p_{2}\right) \\
\left(g_{2 *}+H_{1}^{2} g_{1 *} H_{2}^{1}\left(I+\Delta g_{2 *}\right)\right) \dot{p}_{1}= \\
\tilde{T}_{1}^{2,2} g_{2}+H_{1}^{2} g_{1 *}\left(\dot{\Delta} g_{1}-\tilde{T}_{2}^{1,1} p_{1}\right)
\end{array}\right.
$$

where $\tilde{T}_{2}^{1,1}=-H_{2}^{1} \tilde{T}_{1}^{2,2} H_{1}^{2}$ can be any relative twist of the two bodies and $\Delta>\Delta_{\min }$ for some $\Delta_{\min }<0$ depending on the surfaces and $\Delta$ is defined as in (7).

Proof We first compute the time derivative of $\Delta$, e.g. the change of distance between the bodies.

$$
\begin{aligned}
\dot{\Delta} & =\left\langle\dot{g}_{1}, H_{2}^{1} p_{2}-p_{1}\right\rangle+\left\langle g_{1}, \dot{H}_{2}^{1} p_{2}+H_{2}^{1} \dot{p}_{2}-\dot{p}_{1}\right\rangle \\
& =\left\langle g_{1}, \dot{H}_{2}^{1} p_{2}\right\rangle \\
& =\left\langle g_{1}, \tilde{T}_{2}^{1,1} H_{2}^{1} p_{2}\right\rangle
\end{aligned}
$$

where (9) results since the normal vector $g_{1}$ is always perpendicular to the velocities $\left(\dot{p}_{1}\right.$ and $\left.H_{2}^{1} \dot{p}_{2}\right)$ of the contact points over the surface and since $\dot{g}_{1}$ is perpendicular to $H_{2}^{1} p_{2}-p_{1}$, and (10) results by applying (1).

Using this result for $\dot{\Delta}$, we can compute the time derivative of (6) to obtain the kinematics equation:

$$
\begin{gathered}
\left\{\begin{array}{c}
\dot{p}_{1}+\dot{\Delta} g_{1}+\Delta g_{1 *} \dot{p}_{1}=\dot{H}_{2}^{1} p_{2}+H_{2}^{1} \dot{p}_{2} \\
g_{1 *} \dot{p}_{1}=-\dot{H}_{2}^{1} g_{2}-H_{2}^{1} g_{2 *} \dot{p}_{2}
\end{array}\right. \\
\left\{\begin{array}{c}
\dot{p}_{2}=H_{1}^{2}\left(\dot{p}_{1}+\dot{\Delta} g_{1}+\Delta g_{1 *} \dot{p}_{1}-\dot{H}_{2}^{1} p_{2}\right) \\
g_{1 *} \dot{p}_{1}=\tilde{T}_{2}^{1,1} g_{1}-H_{2}^{1} g_{2 *} \dot{p}_{2}
\end{array}\right.
\end{gathered}
$$

If we now substitute the first equation of (11) into the second, and repeat the whole derivation with objects 1 and 2 switched, we immediately obtain (8). Note that for $\Delta \equiv 0$, we recover the regular contact kinematics (5).

Now consider again the requirements for a unique solution $\dot{p}_{1}$. First we look at the term $\dot{\Delta} g_{2}-\tilde{T}_{1}^{2,2} p_{2}$ in (8) and take the inner product with $g_{2}$ :

$$
\begin{aligned}
\left\langle g_{2}, \dot{\Delta} g_{2}-\tilde{T}_{1}^{2,2} p_{2}\right\rangle & =\dot{\Delta}-\left\langle H_{2}^{1} g_{2}, H_{2}^{1} \tilde{T}_{1}^{2,2} p_{2}\right\rangle \\
& =\dot{\Delta}-\left\langle g_{1}, \tilde{T}_{2}^{1,1} H_{2}^{1} p_{2}\right\rangle \\
& =0
\end{aligned}
$$

where we used (6), (10), and the fact that a homogeneous transformation preserves the inner product. This shows that $\dot{\Delta} g_{2}-\tilde{T}_{1}^{2,2} p_{2}$ is always tangent to the surface, so the righthand side of (8) is well-defined for all twists $T_{1}^{2,2}$.

Whether $\left(g_{1 *}+H_{2}^{1} g_{2 *} H_{3}^{2}\left(I+\Delta g_{1 *}\right)\right)$ has rank two cannot be easily related to properties of the objects, since it also depends on the distance $\Delta$. Even though an object may be relative convex (i.e. the contact points vary smoothly as the objects roll over each other), the contact points can jump when the objects are not in contact and move at a certain distance from each other. However, if the objects are absolutely convex, then invertibility is ensured for any $\Delta>\Delta_{\min }$ for some $\Delta_{\min }<0$, i.e. for any positive distance, and for small enough penetrations, where $\Delta_{\min }$ is the largest distance for which the matrix has rank less than two.

Although the kinematic equation (8) is similar to the results obtained in [7], the approach we used here does not depend on extra coordinates and orthogonal parameterization, and is therefore more transparent and easier to interpret and understand geometrically.

\section{Generalized Contact Dynamics}

In this section, we briefly review the concept of a lumped parameter model of compliant contact based on a spatial spring (as discussed in more detail in [4]) to show the modeling application of the kinematics as derived in the previous section. In the modeling process, we assume that the collision between the two objects is partially elastic; the energy stored during the collision is modeled as a spatial spring, and the energy dissipated during the collision is modeled as a damper. Since the damper is trivial to model (even if geometric), we only look at the spring in this section. Figures 2 and 3 illustrate the intuitive idea.
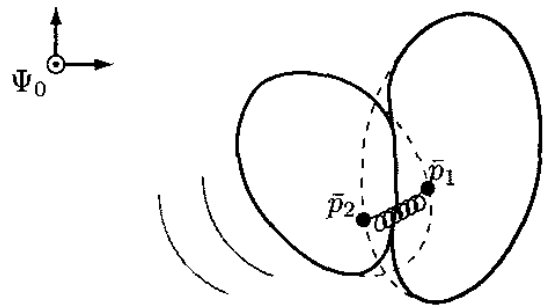

Figure 3: Schematic view of the spring representing the compliant contact as it is attached between the points $\bar{p}_{1}$ and $\bar{p}_{2}$ on the un-deformed bodies.

Starting from a no-contact situation, the position of the generalized contact points is monitored using the kinematic 
equations (7), (10), and (8). When at some time $t_{1}$ the bodies come into contact ( $\Delta$ crosses zero), we connect a spatial spring [12] between two points $\bar{p}_{1}=p\left(t_{1}\right)$ and $\bar{p}_{2}=$ $p\left(t_{1}\right)$ fixed with body 1 and body 2 respectively, in such a way that the initial center of stiffness is at the relative position and orientation of the bodies at impact.

After the impact, the objects continue to move, and the surfaces of the objects deform. We model this in the following way: the points $\bar{p}_{1}$ and $\bar{p}_{2}$ move with the objects as if the ideal, rigid shapes are maintained and penetrate each other. This means that $\bar{p}_{1}$ and $\bar{p}_{2}$ move away from each other, and the spring is charged.

Under the influence of external forces and contact (spring/damper) forces, the objects will start to slide and roll over each other. However, we do not want all movement of the bodies to charge the spring: pure rolling of two bodies over each other is not supposed to charge the spring, since no potential energy is stored in that case. On the other hand, when the bodies are sliding over each other (and in this way locally stretching the surfaces), we do want the spring to be charged, so that potential energy is stored.

The problem is that it is not so easy to distinguish between rolling and sliding if the bodies share an area of contact. In case of point contact, it would have been easy: rolling is when one body rotates relative to the other body around an axis in the tangent plane to the surfaces at the contact point, and sliding is in all other circumstances.

In the case of area contact, we cannot simply talk about the tangent plane to the surface. However, we can talk about the tangent planes at the points $p_{1}$ and $p_{2}$ of maximum penetration depth: these are completely specified by the normal vectors to the surfaces, i.e. by the Gauss maps. By (6) these tangent planes are parallel, and the 'average tangent plane' can be taken as a third parallel plane in between these two. The exact position of this plane depends on the relative stiffnesses of the two objects. We use this average tangent plane to decompose the twist in rolling and sliding, and then use the sliding component to define the spatial spring as attached between $\bar{p}_{1}$ and $\bar{p}_{2}$.

\section{Simulations}

We implemented the 3D kinematics and dynamics model in the simulation package $20 \mathrm{sim}$ [13] and simulated the dynamics of two ellipsoids bouncing on each other and on the floor under the influence of gravity. Since there are three objects, we need to have three copies of the contact model (one between each pair of objects) to be able to model all contact situations. Figure 4 shows a 2D schematic setup of the model. The sub-models are implemented using screw bond graphs $[14,10]$, which allow for easy modeling of the power ports to capture the energy balance of the system. We use relatively soft settings for the spring to be able to see more clearly what happens.

We drop the two ellipsoids at some distance right above each other, with zero initial velocity. Figures 5 and 6 show the results, indicating also the following time instants in the simulation:

(a) The two objects start from a certain height, with some distance between them, the largest distance is between the black ellipsoid and the ground.

(b) The grey ellipsoid hits the ground first and compresses a bit. When the black ellipsoid hits the grey, the grey

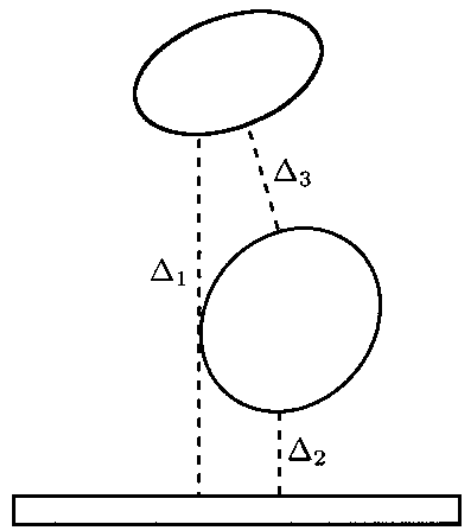

Figure 4: Schematic setup of the simulation model. We use three copies of the contact model to model all possible collisions between the three objects.

is penetrated more into the ground.

(c) The two ellipsoids start to roll over each other.

(d) As the black ellipsoid rolls over the grey, it approaches the ground fast.

(e) The black ellipsoid touches the ground.

(f) Both ellipsoids roll away, creating a distance between them.

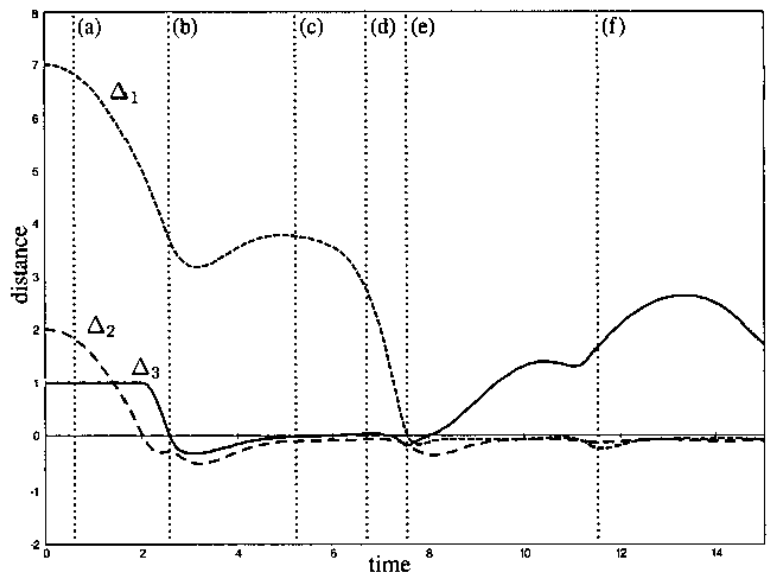

Figure 5: Time evolution of the distances between the three objects. The labels $\Delta_{1}, \Delta_{2}, \Delta_{3}$ correspond to the labels in Figure 4, and the labels (a) through $(f)$ correspond to the labels in Figure 6.

\section{Conclusions and Future Work}

In this paper, we derived the kinematic equations for regular and generalized contact. Starting from simple geometric equations that describe the properties of the contact points, 

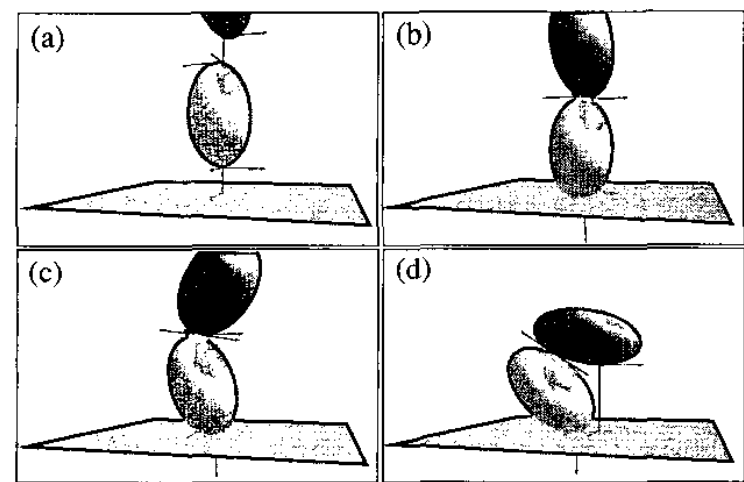

(d)
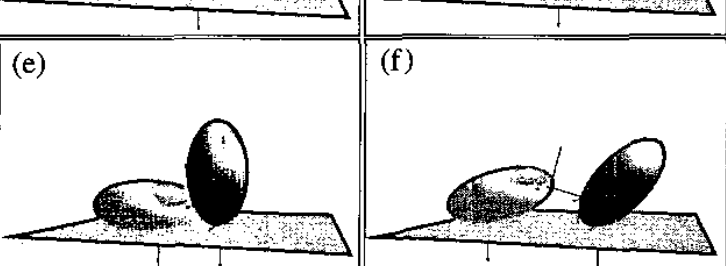

Figure 6: Snapshots of the simulation of two ellipsoids bouncing on each other and on the floor. The labels (a)(f) correspond to the labels in Figure 5. The plots also show the contact frames, attached to the generalized contact points at each object.

we obtained the kinematic equations in very few steps. We briefly reviewed earlier work on the dynamics of contact that uses the kinematic results, and then showed a simulation of the contact model in action.

The model shows good behavior in terms of physical intuition: the bouncing of the objects on each other looks natural. However, the parameters that describe he compliance of the contact are still hand-picked. The next step in the modeling process would be to study practical results in contact modeling (e.g. Pacejka [15] is well-known for practical models of car tires) to obtain realistic parameter values that capture the behavior of real-life materials.

Another important aspect that needs to be added to the model is slip. In practice, the contact forces cannot grow infinitely large, but at a certain value the contact is broken and the objects slide freely over each other. This aspect is also very important for control, since it limits the forces the robot can exert on the ground and therefore restricts the set of suitable controllers for the robot in these circumstances; walking on sand may require different control than walking on tarmac.

Finally, we assumed in the kinematics analysis that the objects were (absolutely) convex, since this ensures that the contacts points move continuously over the surface. It would be interesting to see how the results could be applied to non-convex objects, for example by approximating these objects by a finite set of convex objects and comparing the distances of this set of possible contact points to obtain the real contact point as the minimum of this finite set.

The contact model described in this paper will be used in the 3D modeling of a walking robot to describe the contact of the feet with the soil. We hope it will help us in the analysis and design of controllers that stabilize the robot in walking and make it robust against variations in the soil.

\section{Acknowledgment}

This work has been done in the context of the European sponsored project GeoPlex with reference code IST-2001-34166. Further information is available at http://www.geoplex.cc.

\section{References}

[1] T. McGeer, "Passive bipedal running," in Proc. $R$. Soc. Lond. B240, 1990, pp. 107-134.

[2] D.E. Koditschek and M. Bühler, "Analysis of a Simplified Hopping Robot," The International Journal of Robotics Research, vol. 10, no. 6, pp. 587-605, December 1991.

[3] M. Garcia, A. Chatterjee, A. Ruina, and M. Coleman, "The Simplest Walking Model: Stability, Complexity, and Scaling," ASME Journal of Biomechanical Engineering, vol. 120, no. 2, pp. 281-288, April 1998.

[4] S. Stramigioli and V. Duindam, "A Novel Lumped Spatial Model of Tire Contact," in Proceedings of the IEEE International Conference on Intelligent Transportation Systems, 2002.

[5] David J. Montana, "The Kinematics of Contact and Grasp," International Journal of Robotics Research 7(3), pp. 17-32, 1989

[6] David J. Montana, "The Kinematics of Contact with Compliance," in Proceedings of the IEEE Conference on Robotics and Automation, 1989, pp. 770-774.

[7] M. Visser, S. Stramigioli, and C. Heemskerk, "Screw Bondgraph Contact Dynamics," in Proceedings of the IEEE/RSJ International Conference on Intelligent Robots and Systems, 2002.

[8] Arjan van der Schaft, $L_{2}$-Gain and Passivity Techniques in Nonlinear Control, Springer-Verlag, 2000.

[9] R.M. Murray, Z. Li, and S.S. Sastry, A Mathematical Introduction to Robotic Manipulation, CRC Press, 1994.

[10] S. Stramigioli, Modeling and IPC Control of Interactive Mechanical Systems - A Coordinate-Free Approach, Springer-Verlag, 2001.

[11] R. Gilmore, Lie Groups, Lie Algebras, and Some of Their Applications, John Wiley.\& Sons, 1974.

[12] S. Stramigioli and V. Duindam, "Variable Spatial Springs for Robot Control Applications," in Proceedings of the IEEE/RSJ International Conference on Intelligent Robots and Systems, 2001, pp. 1906-1911.

[13] Control Lab Products, "20sim version 3.2," http://www.20sim.com, 2002.

[14] H.M. Paynter, Analysis and Design of Engineering Systems, M.I.T. Press, 1961.

[15] H.B. Pacejka, Tyre Factors and Vehicle Handling, Delft University of Technology, 1978. 